Revista de Ciencias Sociales - Número 70 (2017) - Páginas 75-94

Discriminación: el caso de Lorenza Cayuhán

\title{
DISCRIMINACIÓN: EL CASO DE LORENZA CAYUHÁN
}

\author{
DISCRIMINATION: THE CASE \\ OF LORENZA CAYUHÁN
}

PATRICIA PÉREZ GOLDBERG*
Universidad de Valparaíso (Chile)
pperezgoldberg@icloud.com

\section{Resumen}

El artículo se refiere a la sentencia de la Corte Suprema chilena que acogió un recurso de amparo en favor de Lorenza Cayuhán, embarazada privada de libertad, para cuyo traslado se aplicaron medidas de seguridad que se prolongaron hasta el momento del parto. Se destaca la utilización del concepto de discriminación "interseccional” para fundamentar la decisión en favor de la peticionaria. Se plantea que este caso permite ilustrar la necesidad de incorporar la perspectiva de género en el ámbito penitenciario.

\section{Palabras clave}

Discriminación interseccional, mujeres encarceladas, perspectiva de género, política penitenciaria.

* Abogada, Licenciada en Ciencias Jurídicas por la Universidad de Valparaíso. Magíster en Derecho Penal y Ciencias Penales por la Pontificia Universidad Católica de Valparaíso, Doctora honoris causa por la California Western School of Law y doctoranda en Derecho por la Universidad de Valparaíso. Artículo recibido el 17 de mayo de 2017 y aceptado el 3 de julio de 2017.

Revista de Ciencias Sociales - Número 70 (2017) - Universidad de Valparáíso - ISSN 0716-7725-Valparaíso, Chile 


\begin{abstract}
The article refers to the Chilean Supreme Court ruling that granted an habeas corpus petition in favor of Lorenza Cayuhán, an incarcerated pregnant woman, that was transported under security measures that lasted until the moment of childbirth. It emphasizes the use of the concept of "intersectional" discrimination to support the decision in favor of the petitioner. It is argued that this case illustrates the need to incorporate a gender perspective in the penitentiary field.
\end{abstract}

\title{
Keywords
}

Intersectional discrimination, women in prison, gender perspective, penitentiary policy.

\section{Introducción}

El caso de Lorenza Cayuhán ofrece la posibilidad de analizar el trato dispensado por agentes del Estado a una mujer embarazada, perteneciente a la etnia mapuche, que se encontraba privada de libertad.

Conociendo de una apelación, el 1 de diciembre de 2016 la Corte Suprema ${ }^{1}$ (en adelante, la Corte) acogió el amparo interpuesto en favor de Lorenza Cayuhán, el cual había sido desestimado por la Corte de Apelaciones de Concepción. La Corte determinó que el uso de medidas de coerción en el traslado y parto de la reclusa, había constituido un trato degradante y violento por parte de los funcionarios penitenciarios, configurándose un acto de discriminación en contra de Lorenza, en su condición de mujer.

El problema planteado es relevante pues permite advertir la particular situación de vulnerabilidad en la que se encuentran las mujeres encarceladas. La condición de gestante es uno de los múltiples factores que hacen diferente la experiencia de privación de libertad para las mujeres y que requieren la adopción de medidas especiales a su respecto.

Se postulará que es indispensable y urgente incorporar una perspectiva de género en el ámbito penitenciario, dando pleno cumpli-

1. Causa Rol 92.795-146.

Facultad de Derecho y Ciencias Sociales - Universidad de Valparaíso - Chile 
miento a las disposiciones del Derecho Internacional de los Derechos Humanos y, en particular, adecuando nuestro ordenamiento normativo a las Reglas de Bangkok.

Este artículo se divide en cuatro partes. En la primera, se describirán los aspectos centrales de la sentencia. En la segunda, se explicará el problema jurídico al que se enfrenta el fallo. En la tercera, se analizará críticamente la resolución a la luz de la obligación de control de convencionalidad, el supuesto de interseccionalidad en la discriminación y la necesidad de adoptar una perspectiva de género en el contexto penitenciario. Por último, se avanzarán algunas conclusiones.

\section{Aspectos centrales de la sentencia}

El 13 de octubre del año 2016 Lorenza Cayuhán tenía 32 semanas de embarazo y estaba cumpliendo una condena en el Centro de Detención Preventiva de Arauco. Ese día, cerca de las 14 horas, dos funcionarios de Gendarmería (un hombre y una mujer) la llevaron al Servicio de Urgencia de la misma ciudad, a bordo de un taxi particular. Escoltaban a dicho vehículo, un carro institucional en el que se desplazaban cinco gendarmes y adicionalmente, dos motoristas de Carabineros. En dicho recinto asistencial se le diagnosticó preeclampsia y a las 18 horas fue trasladada en ambulancia al Hospital Regional de Concepción, siendo custodiada por dos funcionarios de Gendarmería quienes durante el trayecto (de aproximadamente una hora) la mantuvieron "engrilletada por el pie izquierdo a la camilla de la ambulancia" (Considerando $\mathrm{N}^{\circ}$ 5). Al ingresar al Hospital Regional fue evaluada en presencia de una funcionaria de Gendarmería, para lo cual se le habrían retirado los grilletes a petición del personal médico. Éstos se los volvieron a poner cerca de las 22 horas.

El 14 de octubre alrededor de las 15 horas, Lorenza fue trasladada a la Clínica de la Mujer de Concepción, lugar donde — a petición del personal de salud-, se le retiran las medidas de seguridad para realizar el respectivo monitoreo, las que no son repuestas. Durante dicha evaluación, permaneció en la sala una funcionaria de Gendarmería quien a las 16 horas presenció el nacimiento de la pequeña Sayén. La

Revista de Ciencias Sociales - Número 70 (2017) - Universidad de Valparáíso - ISSN 0716-7725-Valparáiso, Chile 
madre fue trasladada a la Clínica Sanatorio Alemán, pues la Clínica de la Mujer no contaba con Unidad de Cuidados Intensivos. (Considerando $\mathrm{N}^{\circ}$ ).

En su sentencia la Corte estableció que Gendarmería de Chile había incurrido en una serie de infracciones a la normativa nacional e internacional a la que Chile está obligado en el tratamiento de las personas privadas de libertad y, en particular, de mujeres en estado de gravidez.

En el ámbito nacional, la Corte estimó transgredido el artículo 1 de la Ley Orgánica de Gendarmería, en cuanto la finalidad de este organismo "es atender, vigilar y contribuir a la reinserción social de las personas privadas de libertad". Asimismo, entendió vulneradas un conjunto de normas del Reglamento de Establecimientos Penitenciarios, referidas fundamentalmente al vínculo de derecho público que existe entre los internos y el Estado, a la prohibición de torturas, tratos crueles, inhumanos o degradantes y a la obligación de que el régimen de las personas privadas de libertad se sujete a la Constitución, tratados internacionales ratificados por Chile y a las leyes y reglamentos pertinentes.

En el plano internacional, la Corte determinó la infracción a disposiciones del Pacto Internacional de Derechos Civiles y Políticos, de la Convención Americana sobre Derechos Humanos (en adelante, $\mathrm{CADH}$ ), de la Convención Interamericana para Prevenir, Sancionar y Erradicar la Violencia contra la Mujer, de la Convención sobre la Eliminación de todas las formas de Discriminación sobre la Mujer (en adelante, CEDAW por sus siglas en inglés), como también el incumplimiento de determinados artículos de las Reglas Mínimas de las Naciones Unidas para el tratamiento de los reclusos (Reglas de Tokio) y de las Reglas de las Naciones Unidas para el Tratamiento de las reclusas y medidas no privativas de la libertad para las mujeres delincuentes (Reglas de Bangkok).

Concretamente, la Corte estableció dos vulneraciones esenciales. En primer lugar, estimó que Gendarmería había lesionado la dignidad de la amparada mediante conductas constitutivas de un trato degradante. Dicho trato se materializó, por una parte, en la aplicación de medidas de coerción innecesarias "[...] no resultaba admisible en el caso de

Facultad de Derecho y Ciencias Sociales - Universidad de Valparaíso - Chile 
marras el uso de grilletes en contra de la amparada, atendido que su traslado de urgencia desde la unidad penal a los distintos recintos de salud a que fue conducida, obedeció a su estado de gravidez y a la inminencia de un parto complejo desde el punto de vista médico, constituyendo el uso de grilletes una forma de represión y sujeción y, por ende, de coerción, que resultaba improcedente [...]" (Considerando $\left.\mathrm{N}^{\circ} 11\right)$ y por otra, en la circunstancia de haber sido custodiada por funcionarios de Gendarmería hasta en el momento mismo del parto, sin que se adoptaran medidas menos invasivas que pudieran conjurar un improbable peligro de fuga,

En segundo lugar, determinó que la institución penitenciaria había atentado contra el derecho a vivir una vida libre de violencia, señalando la Corte que "el Estado ha transgredido la obligación de proteger a la amparada de la violencia ejercida por funcionarios de Gendarmería, al permitir que aquella que se encontraba una especial condición de vulnerabilidad, dado su estado de embarazo y su privación de libertad, fuera sometida a tratos vejatorios que debieron evitarse" (Considerando $\left.\mathrm{N}^{\circ} 12\right)$.

En síntesis, para la Corte, estas afectaciones configuraron un acto de discriminación contra Lorenza Cayuhán en su condición de mujer (Considerando $\left.\mathrm{N}^{\circ} 14\right)$.

Por lo anterior, cuatro fueron las medidas ordenadas a Gendarmería para restablecer el imperio del derecho y asegurar la debida protección de la recurrente: (Considerando $\mathrm{N}^{\circ} 17$ ).

1. "La custodia de la amparada y las medidas de seguridad que se adopten [...] durante los traslados de aquélla a algún recinto asistencial de salud se efectuarán dando estricto cumplimiento a lo previsto en las Reglas 47, 48 y 49 de la Reglas mínimas de las Naciones Unidas para el tratamiento de los reclusos".

2. "Durante dichos traslados, así como durante su permanencia en dichos recintos su custodia directa será ejercida exclusivamente por personal femenino".

3. La institución penitenciaria "deberá revisar y adecuar sus protocolos de actuación en materia de traslado a hospitales externos, conforme a la normativa internacional suscrita por Chile relativa a mujeres privadas de libertad, embarazadas o con hijos lactantes, así

Revista de Ciencias Sociales - Número 70 (2017) - Universidad de Valparáíso - ISSN 0716-7725-Valparaíso, Chile 
como a aquella relativa a la erradicación de todas las formas de violencia y discriminación en contra de las mujeres”.

4. Gendarmería "deberá remitir copia de los resultados del sumario administrativo que lleva adelante con motivo de estos hechos a la Ilustrísima Corte de Apelaciones de Concepción, dentro de un plazo no superior a 30 días, además de informar a dicho tribunal sobre las medidas adoptadas en cumplimiento de los tres puntos precedentes".

\section{Interseccionalidad en la discriminación}

Como he señalado, la Corte estimó que el procedimiento de traslado y custodia de Lorenza Cayuhán había constituido una actuación discriminatoria por parte de los agentes del Estado.

La Corte identifica los factores que confluyeron para la calificación de este trato como discriminatorio, llegando a configurar una "situación paradigmática de interseccionalidad en la discriminación" (Considerando $\mathrm{N}^{\circ}$ 16). En efecto, los funcionarios de Gendarmería al desarrollar tal procedimiento no tomaron en cuenta que la persona trasladada era una mujer con un embarazo de 32 semanas, a punto de dar a luz. Por otra parte, sí tuvieron en consideración su condición de "comunera mapuche" para desplegar un amplio dispositivo de seguridad. Así, concurrían en la especie, dos categorías sospechosas de discriminación.

En cuanto a la primera circunstancia, esto es, el embarazo, vale la pena detenerse brevemente para dimensionar el abismo que separa el marco normativo aplicable (entendido como bloque de constitucionalidad) y la práctica en cuestión. Este caso no versa sobre una conducta aislada que transgredió las regulaciones existentes, sino que existía un instructivo institucional manifiestamente contrario a las mismas, especialmente a las Reglas de Bangkok). Estas disposiciones no sólo complementan las Reglas de Tokio, sino que contienen normas específicas relativas a las mujeres privadas de libertad embarazadas y puérperas. Tal estatuto especial constituye una acción afirmativa en favor de las mujeres encarceladas, en tanto establece en su favor un trato diferenciado orientado a corregir o aminorar la situación de

Facultad de Derecho y Ciencias Sociales - Universidad de Valparaíso - Chile 
desventaja en la que éstas se encuentran. En los términos de Figueroa, se trata de normas que buscan tutelar la igualdad por diferenciación ${ }^{2}$.

Pues bien, el Oficio No 202/2015 del 20 de mayo de 2015 de Gendarmería de Chile, señalaba "se tendrá por regla general una estricta aplicación de medidas de seguridad, a todas las personas que por resolución de autoridades competentes, fueren detenidas o privadas de libertad y que se encuentren hospitalizadas en recintos externos a los institucionales. De manera excepcional, el encargado de custodia considerará la posibilidad de no aplicar alguna de estas medidas de seguridad o retirarlas en su caso, cuando su uso se considere un riesgo a la integridad física y psíquica del interno hospitalizado lo cual procederá sólo cuando el médico tratante o de turno del recinto hospitalario lo solicite por escrito a los funcionarios de custodia, facultativo que deberá argumentar la solicitud en el informe emitido, en caso contrario, cuando no se indique y argumente los motivos de salud, el funcionario de custodia no deberá acceder a lo solicitado, comunicando de manera inmediata al oficial de guardia de la unidad, con el objeto que éste a su vez, lo informe a la jefatura de la unidad para mejor resolver. En los casos de urgencia de salud de un interno, podrá el funcionario de custodia obviar la solicitud escrita de retiro de las medidas de seguridad, pudiendo hacerlo de inmediato, siempre y cuando el propio funcionario de custodia evalúe y determine que las condiciones del entorno sean apropiadas para dicho fin. En caso de cirugías, partos u otros que obliguen el ingreso de interno a pabellón quirúrgico bastará con el requerimiento verbal del médico".

Siguiendo a Figueroa, el mencionado instructivo reflejaría una política de igualdad formal que produce un efecto de discriminación indirecta contra las mujeres embarazadas. Es decir, la regulación en sí misma no implica un trato discriminatorio o diferenciado, pero su aplicación a este grupo produce tal consecuencia.

El Tribunal Constitucional chileno ha definido este tipo de discriminación como aquella que se produce "cuando una ley, una

2. FIGUEROA, Rodrigo: "Igualdad y discriminación”, 2015. Pág. 8. Disponible en: https://www.researchgate.net/publication/268378069_IGUALDAD_Y_ DISCRIMINACION [Fecha de última consulta 1 de mayo de 2017].

Revista de Ciencias Sociales - Número 70 (2017) - Universidad de Valparáíso - ISSN 0716-7725-Valparáiso, Chile 
política o un programa que parecen neutros (por ejemplo en lo que atañe a hombres y mujeres) tienen un efecto discriminatorio en el momento de su aplicación. En ese caso, el resultado o efecto final provoca una situación de desventaja de la mujer con respecto al hombre, debido a las desigualdades existentes no tratadas por una medida supuestamente neutra"'. De la misma manera, la Corte Interamericana de Derechos Humanos, ha hecho mención a este concepto en el caso Nadege Dorzema y otros vs. República Dominicana (2012), a propósito de la expulsión de un grupo de extranjeros indocumentados, la Corte señala que "una violación del derecho a la igualdad y no discriminación se produce también ante situaciones y casos de discriminación indirecta reflejada en el impacto desproporcionado de normas, acciones, políticas o en otras medidas que, aún cuando sean o parezcan ser neutrales en su formulación, o tengan un alcance general y no diferenciado, produzcan efectos negativos para ciertos grupos vulnerables" (Párrafo 235).

Se advierte entonces que el procedimiento que se adoptó en este caso no tuvo en cuenta la naturaleza, trascendencia y riesgo del inminente alumbramiento. Como acertadamente trae a colación la Corte, la Recomendación General N 25 del Comité de la CEDAW señala que "un enfoque jurídico o pragmático puramente formal, no es suficiente para lograr la igualdad de facto con el hombre, que el comité interpreta como igualdad sustantiva (...)", en cuanto "no es suficiente garantizar a la mujer un trato idéntico al del hombre. También deben tenerse en cuenta las diferencias biológicas que hay entre las mujeres y los hombres y las diferencias que la sociedad y la cultura han creado. En ciertas circunstancias será necesario que haya un trato no idéntico de mujeres y hombres para equilibrar esas diferencias [...]" (Considerando $\mathrm{N}^{\circ} 14$ ).

El segundo factor de discriminación, la pertenencia a la etnia mapuche, se estimó acreditado en base a la documentación acompañada por la propia institución penitenciaria, que daba cuenta del tránsito de la amparada por diversos establecimientos asistenciales. El protocolo de seguridad "reforzado" aplicado en este caso, fue a todas luces

3. Causa Rol 1710-2010, Considerando Nº 104.

Facultad de Derecho y Ciencias Sociales - Universidad de Valparaíso - Chile 
desproporcionado en relación al tipo de delito por el cual se encontraba cumpliendo condena (un delito contra la propiedad), a su bajo compromiso delictual y a la nula existencia de indicios de fuga por parte de la interna pudiendo explicarse únicamente por tratarse de una "comunera mapuche", como lo consignaban los documentos ponderados por el tribunal.

\section{Análisis crítico de la sentencia}

La decisión de la Corte Suprema en el caso de Lorenza Cayuhán es de extraordinaria importancia. En tal sentido, sólo mencionaré tres aspectos de especial relevancia jurídica: (i) es un ejercicio del control de convencionalidad, (ii) es un ejemplo de identificación de interseccionalidad en la discriminación y por último, (iii) hace patente la necesidad de considerar la perspectiva de género en el ámbito penitenciario.

1. Control de convencionalidad. En síntesis, es una obligación que tiene toda autoridad pública de revisar la compatibilidad de las normas y demás prácticas internas con la $\mathrm{CADH}$, la jurisprudencia de la Corte Interamericana de Derechos Humanos y los demás tratados de los cuales el Estado sea parte. Dependiendo de la autoridad de que se trate, su ejercicio puede implicar la supresión de normas contrarias a la CADH o bien su interpretación conforme a ésta. La Corte hace esto último, precisando "el sentido de los derechos fundamentales, su respeto, el de las garantías que los protegen” (Considerando $\left.N^{\circ} 17\right)$. En el caso de marras, enfatizando la necesidad de aplicar la normativa penitenciaria a la luz de las exigencias del Derecho Internacional de los Derechos Humanos.

2. Interseccionalidad en la discriminación. Como se explicó antes, la circunstancia de que en su actuación Gendarmería no haya tomado en cuenta el avanzado embarazo de Lorenza Cayuhán y sí haya considerado su pertenencia a una etnia, dio lugar a una situación discriminatoria. Como señala Góngora-Mera, la noción de interseccionalidad apunta a la concurrencia simultánea de diversas causas de discriminación que pueden tener un efecto sinérgico, superior a la simple suma de varias formas de ésta, y que combinadas generan un

Revista de Ciencias Sociales - Número 70 (2017) - Universidad de Valparaíso - ISSN 0716-7725-Valparaíso, Chile 
tipo particular de discriminación ${ }^{4}$. Se trata de un encuadre difundido en el ámbito de la criminología feminista, mediante el análisis de factores tales como la raza y el nivel socioeconómico, para alejarse del denominado "esencialismo" esto es, el estudio desde un estereotipo de mujer uniforme (blanca, con recursos económicos, educada, entre otros aspectos). La consideración de estas variables que multiplican la diferencia entre las mujeres infractoras, da cuerpo a este nuevo prisma ${ }^{5}$. Esta posición se ha sostenido hasta el punto de considerar que la criminología tradicional de carácter pretendidamente neutra en términos de género, es decir, aplicable tanto a hombres como mujeres, es en realidad una eminentemente masculina, que tiene como modelo de persona al hombre, y más aún, a uno de determinadas características, proponiendo el surgimiento de la criminología feminista como un imperativo ${ }^{6}$.

Para ilustrar la aplicación práctica de este constructo, mencionaremos dos sentencias. En 2012 el Tribunal Europeo de Derechos Humanos en el caso B.S. vs. España, resolvió implícitamente en torno a esta idea al considerar que "las decisiones dictadas por los órganos jurisdiccionales internos, no tuvieron en cuenta la vulnerabilidad específica de la demandante, inherente a su condición de mujer africana ejerciendo la prostitución. Las autoridades faltaron así a la obligación que les incumbía [...] de adoptar todas las medidas posibles para ver si una actitud discriminatoria hubiera podido, o no, desempeñar algún papel en los sucesos" (Párrafo 71).

Por su parte, en 2015, la Corte Interamericana de Derechos Humanos utilizó esta concepción en el caso que afectó a Talía González

4. GÓNGORA-MERA, Manuel: "Derecho a la salud y discriminación interseccional: una perspectiva judicial de experiencias latinoamericanas". En: Tratado de Derecho a la Salud, L. Clérico, L. Ronconi y M. Aldao, Martín, Buenos Aires, 2013. Pág. 136.

5. BURGUESS-PROCTOR, Amanda: Intersections of race, class, gender, and crime: future directions for feminist criminology. Feminist Criminology, vol. 1, N 1, 2006. Págs. 27-47.

6. FLAVIN, Jeanne: "Feminism for the mainstream criminologist: an invitation". En: Journal of Criminal Justice, vol. 29, 2001. Págs. 271-285.

Facultad de Derecho y Ciencias Sociales - Universidad de Valparaíso - Chile 
Lluy, quien a los tres años de edad recibió una transfusión de sangre contagiándose de VIH. Ello redundó no sólo en la afectación de su salud sino también en que fue estigmatizada e impedida de asistir a la escuela, entre otras graves consecuencias que sufrieron tanto ella como su familia. En su sentencia desfavorable a Ecuador, la Corte estimó que "[...] confluyeron en forma interseccional múltiples factores de vulnerabilidad y riesgo de discriminación asociados a su condición de niña, mujer, persona en situación de pobreza y persona con VIH. La discriminación que vivió Talía no sólo fue ocasionada por múltiples factores, sino que derivó en una forma específica de discriminación que resultó de la intersección de dichos factores, es decir, si alguno de dichos factores no hubiese existido, la discriminación habría tenido una naturaleza diferente [...]" (Párrafo 290).

Cabe mencionar que el actual foco doctrinario en esta materia ha estado puesto más que en identificar grupos o subgrupos de personas víctimas de discriminación, en analizar las estructuras de poder cuya confluencia genera condiciones de mayor vulnerabilidad ${ }^{7}$. Este es también el enfoque que puede apreciarse en la CEDAW y en la Convención de los Derechos de las Personas con Discapacidad ${ }^{8}$.

3. Perspectiva de género. No obstante la evidencia demuestra que es conveniente — con miras a la reducción de la reincidenciautilizar mecanismos distintos al encarcelamiento ${ }^{9}$, lo cierto es que el recurso a la privación de libertad ha ido en sostenido aumento durante los últimos quince años.

El problema radica en que, en mayor o menor medida, los sistemas penitenciarios no se encuentran preparados para cumplir el

7. CHO, Sumi, WILLIAMS CRENSHAW, Kimberlé y MCCAL, Leslie: Toward a Field of Intersectionality Studies: Theory, Applications and Praxis, 38 Signs, 2013. Pág. 797.

8. Para una visión panorámica sobre este desarrollo, consultar el Reporte de la Comisión Europea "Intersectional Discrimination in EU Gender Equality and Non Discrimination Law” (2016).

9. “La reincidencia en el sistema penitenciario chileno" (2012), Fundación Paz Ciudadana.

Revista de Ciencias Sociales - Número 70 (2017) - Universidad de Valparaíso - ISSN 0716-7725-Valparaíso, Chile 
objetivo en el que teóricamente dicen inspirarse: la reinserción social ${ }^{10}$.

A nivel mundial, las mujeres encarceladas constituyen una minoría vulnerable que ha resultado largamente invisibilizada ${ }^{11}$. A nuestro modo de ver, se trata de un grupo cuyas carencias y rezago permiten apreciar con nitidez lo que Zúñiga denomina el "carácter sexuado de la ciudadanía"12.

La infraestructura, la gestión penitenciaria, los programas de educación, capacitación y los espacios laborales han sido diseñados con una visión androcéntrica, que escasamente considera las historias vitales de las mujeres y sus necesidades.

La evidencia nacional en torno a la caracterización de las mujeres privadas de libertad es escasa, pero concordante en destacar las condiciones de marginalidad, exclusión y vulnerabilidad que éstas presentan ${ }^{13}$.

De acuerdo a la Lista Mundial de Encarcelamiento Femenino (julio 2015) hay 700.000 mujeres privadas de libertad en el mundo. En el $80 \%$ de los sistemas penitenciarios las mujeres representan entre un $2 \%$ y un $9 \%$ de la población penal y desde el año 2000 hasta la fecha del reporte, el número de mujeres y niñas en prisión se ha incrementado aproximadamente un $50 \%$.

10. CUNEO, Silvio: El encarcelamiento masivo, Argentina: Ediciones Didot, 2017. Pág. 104.

11. COVINGTON, Stephanie: "Women in prison: approaches in the treatment of our most invisible population”. En: Women and Therapy Journal, Vol. 21, $\mathrm{N}^{\circ}$ 1, 1998. Pág. 141-155; DEVLIN, Angela: Invisible women. What's wrong with women's prisons, Winchester: Waterside Press, 1998; CARLEN, Pat, y WORRALL, Anne: Analysing women's imprisonment, Comwall: Willan Publishing, 2004. Pág. 12.

12. ZÚNIGA, Yanira: "La construcción de la igualdad de género en el ámbito regional americano”. En: Derechos Humanos de los Grupos Vulnerables, Manual, Barcelona: Red de Derechos Humanos y Educación Superior, 2014. Pág. 180.

13. MORALES, Ana, HURTADO, María, FIGUEROA, Omar, ORTIZ, Paula y POLANCO, Diego: "Estudio sobre los niveles de exclusión social en personas privadas de libertad". En: Fundación Paz Ciudadana y Fundación San Carlos de Maipo, 2015.

Facultad de Derecho y Ciencias Sociales - Universidad de Valparaíso - Chile 
Es interesante notar que el nivel de aumento en el encarcelamiento femenino supera al masculino, si se toma en cuenta que en los últimos 15 años la población penitenciaria mundial se elevó en un $20 \%$, lo que contrasta con el $50 \%$ de crecimiento ya referido.

En Chile, considerando los datos disponibles a octubre de $2016^{14}$, el número total de mujeres recluidas era de 3.658 , lo que corresponde al 8,3\% de la población penitenciaria nacional. En cuanto a los delitos por los cuales se encontraban privadas de libertad, se observa una preponderancia de los delitos vinculados al tráfico de drogas $(47,3 \%)$, seguido por robos y hurtos (23,5\% y un $8,2 \%$ respectivamente). De acuerdo a su nivel educacional, los datos muestran que el $46.3 \%$ tenía educación básica, el 47,9\% educación media, el 3.4\% educación superior y el $2,1 \%$ carecía de instrucción. Finalmente, en cuanto a la cantidad de mujeres recluidas que declaran ser madres, un $86,4 \%$ respondió afirmativamente, no se contó con información respecto del 12,7\% y un $0,9 \%$ respondió negativamente ${ }^{15}$.

No obstante Chile ratificó en 1989 la CEDAW, los esfuerzos institucionales para impulsar políticas públicas en este ámbito han sido insuficientes. En efecto, en 2011 la Asamblea General de las Naciones Unidas formuló las Reglas de las Naciones Unidas para el tratamiento de las reclusas y medidas no privativas de la libertad para las mujeres delincuentes, que vinieron a complementar las Reglas mínimas para el tratamiento de reclusos.

Acogiendo esas recomendaciones y en base a la evidencia nacional disponible, se implementaron un conjunto de medidas tendientes a desarrollar una línea de trabajo específica en este ámbito. Bajo dicha orientación se dictó una ley de conmutación de penas para mujeres que reunieran determinados requisitos, se dispuso la creación de la Unidad de Derechos Humanos en Gendarmería, se convocó a una “Mesa de Política Penitenciaria con Enfoque de Género”, se impulsó el

14. Oficio $\mathrm{N}^{\circ} 1567$ de 14 de noviembre 2016 del Director Nacional de Gendarmería de Chile.

15. MINISTERIO DE JUSTICIA: Política penitenciaria con enfoque de género, Santiago, Chile, 2013.

Revista de Ciencias Sociales - Número 70 (2017) - Universidad de Valparáíso - ISSN 0716-7725-Valparaíso, Chile 
Programa de competencias sociales para mujeres, se concretó la remodelación de la ex-cárcel de San Miguel (que pasó a ser un centro exclusivo para imputadas), se creó el primer Centro de Educación y Trabajo (CET) Semiabierto de Santiago "Talita Kum”, entre otras iniciativas. En este marco de acción se concedió en 2012 el indulto particular a la pastora aymara Gabriela Blas, reconociéndose la vulneración persistente de sus derechos. Lamentablemente, varias de las medidas mencionadas no tuvieron continuidad, no existiendo información pública disponible acerca de la eventual reformulación de las mismas o si se tiene previsto retomar esta línea de trabajo en el futuro. Sin perjuicio de lo expresado, con fecha 7 de junio de 2017 el Ministerio de Justicia convocó a un grupo de expertos para integrar un Comité Asesor para la Reinserción Social ${ }^{16}$, el cual está llamado a formular propuestas en siete ejes ${ }^{17}$, no contemplándose, en un principio, la temática penitenciaria vinculada a mujeres como una línea de trabajo autónoma. Con fecha 7 de julio de 2017 y acogiendo la petición de una de las integrantes del referido Comité, el Ministerio de Justicia convocó a un grupo de académicos y representantes institucionales a formular el plan de trabajo del "subcomité de mujeres en el sistema penitenciario".

\section{Conclusiones}

La política penitenciaria de un país, debiera abordarse como una auténtica política de Estado. Lo dicho, aplicado al tema que nos convoca, implica recorrer dos niveles. El primero supone un trabajo consistente en integrar la normativa nacional e internacional que la regula,

16. Comité Asesor para la Reinserción Social, minuta de antecedentes disponible en http://www.minjusticia.gob.cl/media/2017/06/MINUTA-EJECUTIVA-

COMITÉ-ASESOR-PARA-LA-REINSERCIÓN-SOCIAL-5-de-junio.pdf

17. Los siete ejes se abordarán en los siguientes sub comités: "fortalecimiento del enfoque de derechos humanos; fortalecimiento de la oferta especializada en reinserción social; garantizar el acceso de la población del sistema cerrado a la educación formal; mejorar el acceso a capacitación, trabajo y emprendimiento; formación valórica y ciudadana; fortalecimiento de la institucionalidad para la reinserción social y fortalecimiento del sistema post penitenciario".

Facultad de Derecho y Ciencias Sociales - Universidad de Valparaíso - Chile 
en un paradigma teórico que fundamente políticas de acción afirmativa en relación a las mujeres privadas de libertad. En este sentido, creo que un enfoque orientado al desarrollo de capacidades, particularmente en la visión expuesta por Martha Nussbaum ${ }^{18}$, es una base en la que pueden sustentarse tanto las normas como las políticas públicas orientadas a la inclusión.

El segundo, apunta a verificar la correcta ejecución de las políticas que se elaboren dentro del marco teórico referido en el paso anterior. Para esto es necesario asegurar la concurrencia de las siguientes condiciones: (i) la existencia de un diálogo permanente y participativo entre la academia, los poderes del Estado, las instituciones públicas y privadas incumbentes, los ejecutores de políticas públicas y especialmente, con las personas a quienes éstas se dirigen; (ii) que las decisiones se basen en evidencia; (iii) el seguimiento y publicación de los resultados de las políticas implementadas; y (iv) evaluaciones periódicas que permitan introducir los ajustes necesarios. Las metas de corto, mediano y largo plazo en que se exprese la política pública deberían orientar el actuar del órgano estatal, lo cual contribuiría a evitar su abandono o deficiente ejecución por los gobiernos de turno.

La decisión de la Corte Suprema de acoger el amparo interpuesto, es una manifestación elocuente de la importancia de la interpretación judicial en la protección de los derechos humanos en nuestro país. Me parece que la decisión adoptada es el reflejo de una interpretación teleológica, que - como destacan Valenzuela y Villavicencio citando a Irving - ubica a la Constitución y la experiencia de quienes son su objeto, en el contexto social ${ }^{19}$. Adicionalmente, pienso que esta sentencia da cuenta de un tipo de racionalidad jurídica protectora, que, como señala Muñoz, debiera ir acompañada de la construcción de una disciplina jurídica que dé sustento a una lectura prodiscriminado ${ }^{20}$.

18. NUSSBAUM, Martha: Women and Human Development: The Capabilities Approach. Cambridge University Press, 2001.

19. VILLAVICENCIO, Luis: "La constitucionalización de los derechos sexuales y reproductivos. Hacia una igual ciudadanía para las mujeres”. En: Revista Ius et Praxis, Año 21, No 1, 2015. Pág. 279.

20. MUÑZ, Fernando: "Estándares conceptuales y cargas procesales en el litigio antidiscriminación. Análisis crítico de la jurisprudencia sobre la Ley Zamudio entre 2012 y 2015”. En: Revista de Derecho,Vol XXVIII, N² 2015. Pág. 165.

Revista de Ciencias Sociales - Número 70 (2017) - Universidad de Valparáíso - ISSN 0716-7725-Valparáiso, Chile 


\section{BIBLIOGRAFÍA}

BELTRÁN, María Antonieta: "Criminología feminista. Estado del arte y presencia en Latinoamérica”. En: VI Jornadas de Sociología en la Universidad Nacional de La Plata, Departamento de Sociología, Facultad de Humanidades y Ciencias de la Educación, Universidad Nacional de La Plata, 2010.

BLANCHETTE, Kelley, y BROWN, Shelley: The assessment and treatment of women offenders. John Wiley \& Sons Ltd, 2006.

BLOOM, Barbara, y COVINGTON, Stephanie: Gender-specific programing for female offenders: what is it and why is it important? 1998. Disponible en: http://www.stephaniecovington.com/ assets/ files/13.pdf [Fecha última consulta 1 de mayo de 2017].

BLOOM, Barbara, OWEN, Barbara y COVINGTON, Stephanie: Gender-responsive strategies. Research, practice and guiding principles for women offender. National Institute of Corrections, U.S. Department of Justice, 2003. Disponible en: http://static.nicic.gov/ Library/ 018017.pdf [Fecha de última consulta 1 de mayo de 2017].

BURGUESS-PROCTOR, Amanda: Intersections of race, class, gender, and crime: future directions for feminist criminology. Feminist

Criminology, vol. 1, $\mathrm{N}^{\circ}$ 1, 2006. Págs. 27-47.

BRITTON, Dana M.: Feminism in criminology: engendering the outlaw. Annals of the American Academy of Political and Social Science, vol. 571, 2000. Págs. 57-76.

CÁRDENAS, Ana: Mujeres y Cárcel: diagnóstico de las necesidades de grupos vulnerables en prisión. Universidad Diego PortalesICSO, 2011. Disponible en http://www.icso.cl/wp-content/uploads/ 2012/01/Proyecto-Grupos-Vulnerables-CPF-GIZ-MINJU-ICSO-versi $\% \mathrm{C} 3 \% \mathrm{~B} 3 n$-final-para-p\%C3\%A1gina-web-Diciembre-2011.pdf. [Fecha de última consulta 9 de mayo de 2017].

CARLEN, Pat, y WORRALL, Anne: Analysing women's imprisonment, Willan Publishing, Cornwall, 2004.

CENTRO DE DERECHOS HUMANOS, Universidad Diego Portales: Informe Anual sobre Derechos Humanos en Chile, Ediciones Universidad Diego Portales, Santiago, 2004.

Facultad de Derecho y Ciencias Sociales - Universidad de Valparaíso - Chile 
CHO, Sumi, WILLIAMS CRENSHAW, Kimberlé y MCCALL, Leslie: Toward a Field of Intersectionality Studies: Theory, Applications and Praxis, 38 Signs, 2013. Pág. 797.

CORSTON, Jane: The Corston Report, Home Office, Reino Unido. 2007.

CORTE INTERAMERICANA DE DERECHOS HUMANOS: Caso Artavia Murillo y otros ('fecundación in vitro') vs. Costa Rica. Sentencia de 2012.

CORTE INTERAMERICANA DE DERECHOS HUMANOS:

Caso Nadege Dorzema y otros con República Dominicana (2012).

COVINGTON, Stephanie: "Women in prison: approaches in the treatment of our most invisible population". En: Women and Therapy Journal, vol. 21, $\mathrm{N}^{\circ}$ 1, 1998. Págs. 141-155.

COVINGTON, Stephanie: “The relational theory of women's psychological development: implications for the criminal justice system". En: Female offenders: critical perspectives and effective interventions, Ruth Zaplin (ed.), 2008. Págs. 135-164.

CRIGHTON, David y TOWL, Graham: "Risk assessment". En: Psychology in probation services, BPS Blackwell, 2005.

Págs. 52-66.

CUNEO, Silvio: El encarcelamiento masivo, Ediciones Didot, Argentina, 2017.

DALY, Kathleen: "Feminist perspectives in criminology: a review with gen $\mathrm{Y}$ in mind". En: The Handbook of Criminal Theory, Eugene McLaughlin y Tim Newburn (eds.), SAGE Publications, 2010. Págs. 225-246.

DEVLIN, Angela: Invisible women. What's wrong with women's prisons, Waterside Press, Winchester, 1998.

ENROOS, Rosi: "Mothers in prison: between the public institution and private family relations". En: Child and Family Social Work, vol. 16, 2011. Págs. 12-21.

FIGUEROA, Rodrigo: Igualdad y discriminación, 2015. Disponible en:https://www.researchgate.net/publication/ 268378069_IGUALDAD_Y_DISCRIMINACION. [Fecha de última consulta 1 de mayo de 2017].

Revista de Ciencias Sociales - Número 70 (2017) - Universidad de Valparáíso - ISSN 0716-7725-Valparaíso, Chile 
FIGUEROA, Rodrigo: La acción afirmativa en la jurisprudencia del

Tribunal Constitucional, 2016. Disponible en http://dx.doi.org/ 10.4067/S0718-34372016000200003. [Fecha de última consulta 9 de mayo de 2017].

FLAVIN, Jeanne: "Feminism for the mainstream criminologist: an invitation". En: Journal of Criminal Justice, vol. 29, 2001. Págs. 271-285.

FREITAS, Ana María, INACIO, Ana Risca, y SAAVEDRA, Luisa: "Motherhood in prison: reconciling the irreconcilable". En: The Prison Journal, vol. 96, $\mathrm{N}^{\circ}$ 3, 2016. Págs. 415-436.

GÓNGORA-MERA, Manuel Eduardo: "Derecho a la salud y discriminación interseccional: Una perspectiva judicial de experiencias latinoamericanas”. En: Tratado de Derecho a la Salud, L. Clérico, L. Ronconi y M. Aldao, Martín, Buenos Aires, 2013. Págs. 133-159.

HEIDEHNSOHN, Frances, y GELSTHORPE, Loraine: "Gender and crime”. En: The Oxford Handbook of Criminology, Mike Maguire, Rod Morgan y Robert Reiner (eds.), Oxford University Press, 2011.

HEIDEHNSOHN, Frances, y SILVESTRE, Marisa: "Gender and crime". En: The Oxford Handbook of Criminology, Mike Maguire, Rod Morgan y Robert Reiner (eds.), Oxford University Press, 2012.

EUROPEAN COMMISSION: Intersectional Discrimination in EU Gender Equality and Non Discrimination Law. Disponible en: http://ec.europa.eu/justice/genderequality/document/files/ intersectionality.pdf. [Fecha de última consulta 1 de mayo de 2017].

NUSSBAUM, Martha: Women and Human Development: The

Capabilities Approach. Cambridge University Press, 2001.

NUSSBAUM, Martha: Creating capabilities: The Human Development Approach. The Belknap Press of Harvard University Press, 2011. MORALES, Ana, MUÑOZ, Nicolás, WELSH, Gherman y FÁBREGA, Jorge: "La reincidencia en el sistema penitenciario chileno". En: Fundación Paz Ciudadana, 2012. Disponible en: http://www. pazciudadana.cl/wp-content/uploads/2013/07/2013-0321_reincidencia-en-el-sistema-penitenciario-chileno.pdf [Fecha de última consulta 9 de mayo de 2017].

Facultad de Derecho y Ciencias Sociales - Universidad de Valparaíso - Chile 
MORALES, Ana, HURTADO, María, FIGUEROA, Omar, ORTIZ, Paula y POLANCO, Diego: "Estudio sobre los niveles de exclusión social en personas privadas de libertad”. En: Fundación Paz Ciudadana y Fundación San Carlos de Maipo, 2015.

ONU: Informe del Relator Especial sobre la tortura y otros tratos o penas crueles, inhumanos o degradantes, 2016. Disponible en: http://www.ohchr.org/Documents/HRBodies/HRCouncil/ RegularSession/Session22/A-HRC-22-53_sp.pdfh. [Fecha de última consulta 9 de mayo de 2017].

MINISTERIO DE JUSTICIA: Política penitenciaria con enfoque de género, Santiago, Chile, 2013.

MINISTERIO DE JUSTICIA: Minuta relativa al Comité Asesor para la Reinserción Social, Santiago, Chile, 2017.

MUÑOZ, Fernando: "Estándares conceptuales y cargas procesales en el litigio. Análisis crítico de la jurisprudencia sobre la Ley Zamudio entre 2012 y 2015”. En: Revista de Derecho,Vol XXVIII, $\mathrm{N}^{\circ}$ 2015. Pág. 165.

SCOTTISH GOVERNMENT: Commision on Women Offenders, 2012. Disponible en http://www.gov.scot/About/Review/ commissiononwomenoffenders [Fecha de última consulta 9 de mayo de 2017].

TRACY-MUMFORD, Fran: Women in prison status report, 2000.

VALENZUELA, Eduardo, STUVEN, Ana María, MARCAZZOLO, Ximena y LARROULET, Pilar: El impacto social de la prisión femenina: recomendaciones para una política pública en la materia. Centro de Políticas Públicas UC, Santiago, 2012.

VALENZUELA, Cecilia y VILLAVICENCIO, Luis: "La constitucionalización de los derechos sexuales y reproductivos. Hacia una igual ciudadanía para las mujeres”. En: Revista Ius et Praxis, Año 21, No 1, 2015. Págs. 271-314.

VILLAVICENCIO Miranda, Luis: "La concepción política de la persona y las visiones constitutivas del bien”. En: Ideas y Valores, $\mathrm{N}^{\circ}$ 135, 2007. Págs. 29-49.

VILLAVICENCIO Miranda, Luis: "Algunas críticas a la idea de razón pública rawlsiana”. En: Revista de Derecho de la Pontificia Universidad Católica de Valparaíso, vol. XXXII, 2009. Págs. 533-557. 
VILLAVICENCIO Miranda, Luis: "La relevancia moral de la identidad cultural”. En: Polis, Revista de la Universidad Bolivariana, vol. 9, $\mathrm{N}^{\circ}$ 26, 2010. Págs. 399-420.

VILLAVICENCIO Miranda, Luis: "Privatizando la diferencia: el liberalismo igualitario y el pluralismo cultural". En: Revista de Derecho Universidad Austral de Chile, vol. XXIII, N $1,2010$. Págs. 37-57.

VILLAVICENCIO Miranda, Luis: "La neutralidad liberal como antiperfeccionismo". En: Revista de Derecho Universidad Católica del Norte, año 19 - $\mathrm{N}^{\circ}$ 2, 2012. Págs. 371-391.

VILLAVICENCIO Miranda, Luis: "Delineando al liberalismo auténtico. Las tesis centrales del constructivismo rawlsiano". En: Revista de Derechos Fundamentales Universidad Viña del Mar, $\mathrm{N}^{\circ} 10$, 2013. Págs. 157-190.

WALMSLEY, Roy: "World female imprisonment list. Women and girls in penal institutions, including pre-trial detainees/remand prisoners". En: World Prison Brief-Institute for Criminal Policy Research, 2015. Disponible en http://www.prisonstudies.org/sites/ default/files/resources/downloads/world_female_imprisonment list_third_edition_0.pdf [Fecha de última consulta $9 \overline{\text { de }}$ mayo de 2017]. ZÚÑ̃IGA, Yanira: "La construcción de la igualdad de género en el ámbito regional americano". En: Derechos Humanos de los Grupos Vulnerables, Manual, Red de Derechos Humanos y Educación Superior, Barcelona, 2014. Págs. 179-210. Disponible en https://www.upf.edu/ dhes-alfa/materiales/res/dhgv_pdf/ DHGV_Manual.179-210.pdf [Fecha de última consulta 1 de mayo de 2017].

ZÚÑIGA, Yanira: El derecho al desarrollo en la perspectiva de género. Tesis doctoral, Instituto Bartolomé de las Casas de Derechos Humanos, 2003. Pág. 169.

Facultad de Derecho y Ciencias Sociales - Universidad de Valparaíso - Chile 